\title{
The active rather than intrinsic mechanism of nucleosome de- pletion by poly(dA:dT) tracts
}

Toby Barnes ${ }^{1}$ and Philipp Korber ${ }^{2, *}$

1 Biomedical Center (BMC), Divison of Molecular Biology, Faculty of Medicine, LMU Munich; Toby.Barnes@bmc.med.lmu.de

2 Biomedical Center (BMC), Divison of Molecular Biology, Faculty of Medicine, LMU Munich; pkorber@lmu.de

* Correspondence: pkorber@lmu.de; Tel.: +49-89-218075435

Abstract: Poly(dA:dT) tracts cause nucleosome depletion in many species, e.g., at promoters and replication origins. Their intrinsic biophysical sequence properties make them stiff and unfavorable for nucleosome assembly, as probed by in vitro nucleosome reconstitution. The mere correlation between nucleosome depletion over poly $(\mathrm{dA}: \mathrm{dT})$ tracts in vitro and in vivo inspired an intrinsic nucleosome exclusion mechanism in vivo. However, we compile here published and new evidence that this correlation does not reflect mechanistic causation. 1) Nucleosome depletion over poly(dA:dT) in vivo is not universal, e.g., very weak in S. pombe. 2) The energy penalty for incorporating poly (dA:dT) tracts into nucleosomes is modest $(<10 \%)$ relative to ATP hydrolysis energy abundantly invested by chromatin remodelers. 3) Nucleosome depletion over poly(dA:dT) is much stronger in vivo than in vitro if monitored without MNase and 4) actively maintained in vivo. 5) S. cerevisiae promoters evolved a biased poly (dA) versus poly(dT) distribution. 6) Nucleosome depletion over poly(dA) is directional in vivo. 7) The ATP dependent chromatin remodeler RSC preferentially and directionally displaces nucleosomes towards $5^{\prime}$ of poly $(\mathrm{dA})$. Especially bias and directionality would not be expected for an intrinsic mechanism. Together, this argues for a mechanism where active and species-specific read out of intrinsic sequence properties, e.g., by remodelers, shapes nucleosome occupancy.

Keywords: nucleosome; chromatin; poly(dA:dT); remodeling; RSC;

Nucleosomes are depleted in vivo over poly(dA:dT) tracts in many species

Nucleosome occupancy is prominently low over homopolymeric poly(dA:dT) sequence tracts in a wide variety of species including S. cerevisiae and other yeasts, fly, worm, mouse and human cells [1-3]. Nucleosome depletion scales directly with tract length. Clear effects usually start with five and more dA:dT base pairs (bp) in a row and are increasingly diminished with the number of interruptions in the homopolymeric sequence. As most nucleosome mapping is based on limited digests with Micrococcal Nuclease (MNase) $[4,5]$ and as MNase has a sequence preference for dA:dT-rich sequences $[6,7]$, there is the concern that the depletion of nucleosome signal in MNase-seq and related techniques reflects a technical bias. Indeed, it is recognized since long [8-11] and sometimes explicitly controlled for (e.g., [10-12]) that nucleosomes with dA:dT-rich DNA are depleted faster during MNase digestion kinetics. The depletion over poly(dA:dT) tracts in vivo is mostly (see below) stronger than can be explained by this MNase bias and, more to the point, confirmed, at least for some species like S. cereivisae, in MNase-independent methods like anti-histone ChIP with sonication [11, 13], ChIP-exo [14], chemical mapping $[15,16]$ and DNA methylation footprinting [17-19].

\section{Nucleosome depletion is functionally important and caused by poly(dA:dT) tracts}

Poly(dA:dT) tracts and their associated low nucleosome occupancy is linked to functional genomic regions like promoters [20-24] and replication origins [25]. As the assembly of protein complexes like the transcription or replication machinery on these DNA regions 
would be hindered by nucleosomes, it is functionally important that there are mechanisms that remove nucleosomes from these regions. Poly $(\mathrm{dA}: \mathrm{dT})$ tracts constitute one of these mechanisms as their direct causality was demonstrated in vivo by introduction or removal of such tracts and the corresponding decrease or increase, respectively, in DNA accessibility/nucleosome occupancy [21, 22, 26, 27].

\section{Special intrinsic properties of poly(dA:dT) tracts were suggested to cause nucleosome depletion in vivo by a nucleosome-intrinsic mechanism}

Many biophysical studies established intrinsic properties of poly $(\mathrm{dA}: \mathrm{dT})$ tracts that deviate from those of generic DNA sequences [3]. These properties include a shorter helical repeat, a more narrow minor groove, more extensive hydration, more extensive base overlap within one strand, and lead overall to increased stiffness (higher deformation energy [28]) and higher energetic costs for the wrapping and twisting of DNA around the histone octamer during nucleosome assembly. These costs can be observed and quantified in competitive nucleosome reconstitutions where several DNA sequences compete for nucleosome assembly [29-31]. The classical reconstitution method is salt gradient dialysis (SGD). Purified DNA and histone octamers are initially mixed at high ionic strength, usually $2 \mathrm{M} \mathrm{NaCl}$, where DNA and histones hardly interact. The salt concentration is decreased gradually or in a stepwise way by dialysis or dilution, respectively, so that the electrostatic interactions between DNA and histones [32, 33] begin to drive nucleosome assembly. Due to the slow salt dilution, there is a sufficiently long period during which histones and DNA experience an ionic strength around ca. $1 \mathrm{M} \mathrm{NaCl}$. This salt regime allows repeated nucleosome assembly and disassembly, i.e., the histone octamers, more precisely the (H3-H4)2 tetramers, which assemble first on the DNA, can effectively equilibrate to their energetically preferred positions and avoid unfavourable sequences [34-36]. This procedure was extensively used to compare different DNA sequences for their relative nucleosome formation propensities and to select especially strong nucleosome positioning sequences, like the "Widom 601" sequence [29, 30, 36, 37]. The outcome of such measures is usually referred to as the "intrinsic" nucleosome formation propensity or affinity of a DNA sequence. Poly(dA:dT) tracts score low in this regard [29] and lead to low nucleosome occupancy, for example, in SGD with whole genomes [38-40].

It should be noted that SGD-derived intrinsic properties are measured under nonphysiological conditions as the histones equilibrate to their positions at around $1 \mathrm{M}$ salt and become kinetically stuck at lower salt concentrations, i.e., cannot re-equilibrate to other positions, even if these were the thermodynamically preferred ones at lower salt [30, $33,35,36]$. Incubation of SGD-reconstituted nucleosomes at low salt but elevated temperature, like $40-60^{\circ} \mathrm{C}$, allows histone octamers to move again along the DNA and to re-equilibrate to positions that are preferred under these conditions and that may be different from the positions equilibrated at high salt but lower temperature [41, 42]. This illustrates how intrinsic nucleosome positioning preferences depend on the thermodynamic conditions. Unfortunately, there is no technique available that would allow histone octamers to equilibrate to their intrinsically preferred positions at physiological ionic strength and temperature without inclusion of other non-histone factors, which may again tweak thermodynamic preferences. Therefore, we do not know the physiological purely intrinsic sequence preferences for nucleosome assembly. Intrinsic preferences measured by SGD or similar techniques, like equilibrium measurements at physiological buffer and temperature conditions but in the presence of the histone chaperone Nap1 [43], have to be taken as operational approximations of the in vivo situation [44].

Nonetheless, there is a striking correlation between nucleosome depletion over poly(dA:dT) tracts in vivo and in SGD-reconstituted chromatin in vitro [38-40]. This and alone this correlation has been (mis)taken as evidence that this nucleosome depletion in vivo is mechanistically due to poly $(\mathrm{dA}: \mathrm{dT})$ intrinsically disfavouring nucleosome assembly $[3,45,46]$. This mechanism is called "intrinsic" to signify that only the intrinsic properties of DNA and histone octamer in the nucleosome, without thermodynamic influence of other factors, determine such nucleosome depletion. By principle, correlation need not 
reflect causality and in the following we delineate the evidence against this intrinsic nucleosome depletion mechanism.

\section{In vivo nucleosome depletion over poly $(\mathrm{dA}: \mathrm{dT})$ tracts is not universal}

As canonical histones are among the most highly conserved proteins, it may be expected that the intrinsic nucleosome disfavouring properties of poly $(\mathrm{dA}: \mathrm{dT})$ tracts should play out across species. Indeed, nucleosome depletion over poly(dA:dT) was observed in SGD using histones from several species, e.g., fly or chicken histones [38, 40], and in many species in vivo [1-3]. However, there is the notable exception of $S$. pombe. Here, nucleosome depletion over poly(dA:dT) is far less pronounced than in S. cerevisiae and dA:dT-rich sequences are not enriched in linkers or nucleosome-depleted promoter regions but within nucleosomes close to the dyad $[47,48]$. Nucleosome depletion over poly(dA:dT) as based on MNase-seq measurements for $S$. pombe was not confirmed by chemical mapping [48]. For example, dA:dT-rich replication origins in $S$. pombe appeared nucleosome depleted in MNase-based but not in chemical mapping. This is a case of misleading MNase sequence bias. We wonder if we had ever heard of the intrinsic "genomic code for nucleosome positioning" [49] had S. pombe rather than S. cerevisiae been the main model species.

As another exception from a putative universal intrinsic mechanism, introduction of a $(\mathrm{dA}: \mathrm{dT})_{19}$ tract into a well-positioned nucleosome at the $S$. cerevisiae PHO84 promoter did not lead to removal of this nucleosome like otherwise seen upon promoter activation, but a clear DNaseI footprint was maintained and nuclease accessibility increased only moderately [50].

\section{The energetic penalty for incorporation of poly(dA:dT) into nucleosomes is not very high}

The manipulated $\mathrm{PHO} 84$ promoter nucleosome is a counterexample regarding the misconception that poly $(\mathrm{dA}: \mathrm{dT})$ tracts cannot be incorporated into nucleosomes at all. There are many other examples in vivo where nucleosomes are positioned over poly(dA:dT) tracts, for example by the ATP depedent S. cerevisiae remodeler ISW2 [51] or at (dA:dT)-rich terminator regions in $S$. cerevisiae, which leads to MNase-sensitive nucleosomes [11]. Also in vitro reconstitution of nucleosomes onto poly(dA:dT)-containing sequences is well possible $[52,53]$, including even a crystal structure of a nucleosome with a $(\mathrm{dA}: \mathrm{dT}) 16$ tract [54].

Not only can poly $(\mathrm{dA}: \mathrm{dT})$ tracts be nucleosomal in vitro and in vivo, the respective energetic cost is actually not as high as initially assumed. The more extreme differences between intrinsic affinities of histone octamers to DNA sequences as measured by competitive SGD in the Widom group were $~ 5000$ fold, which corresponds to an energetic difference $(\Delta \Delta \mathrm{G}=\mathrm{RT} \ln 5000)$ at $30^{\circ} \mathrm{C}$ of ca. $5 \mathrm{kcal} / \mathrm{mol}$. This is not even half the energy of one ATP hydrolysis reaction (ca. $12 \mathrm{kcal} / \mathrm{mol}$ under physiological conditions [55]). Nucleosome dynamics are mediated in vivo by ATP dependent chromatin remodeling enzymes (remodelers) [56, 57] that (dis)assemble, reposition (slide) and reconfigure (histone exchange) histone octamers on DNA. For some remodelers a step size of 1-2 bp per ATP hydrolysis was estimated [58-60], which amounts to hundreds of ATP hydrolyses for sliding a nucleosome along DNA even over short distances. Therefore, we suggest that the energetic differences of intrinsic sequence preferences for nucleosome formation are merely ripples in a remodeler-dominated energy landscape and may be easily overcome by ATP input.

Further, reassessement of energetic measurements arrived at quite smaller energetic differences than before. If the $5 \mathrm{~S}$ rDNA positioning sequence was taken as reference point, then the Widom 601 strong positioning sequence showed a difference of $0.7 \mathrm{kcal} / \mathrm{mol}$ ( 3fold higher affinity) in the Nap1-assembly method [61], which is less than the originally reported $2.9 \mathrm{kcal} / \mathrm{mol}$ ( 124 fold higher affinity) derived by competitive SGD. Comparison of mononucleosomes containing poly $(\mathrm{dA}: \mathrm{dT})$ tracts taken from $S$. cerevisiae promoters showed that they are less stable by at most $0.86 \mathrm{kcal} / \mathrm{mol}$ ( 4 fold lower affinity) relative to $5 S$ rDNA-nucleosomes in competitive salt step reconstitutions [53], i.e., by less than 10\% 
of ATP hydrolysis energy. Not that the 5SrDNA reference itself is already a strong nucleosome positioning sequences. Therefore, the energetic differences between poly $(\mathrm{dA}: \mathrm{dT})-$ containing sequences and the average genomic sequences will be even smaller. Nonetheless, this does not preclude that they matter in the end.

\section{Nucleosome depletion over poly(dA:dT) tracts by the intrinsic mechanism in vitro in} SGD is much weaker than by the in vivo mechanism

The caveat that MNase digestion bias may exaggerate the appearance of nucleosome depletion (see above) was not controled for in SGD chromatin by MNase-independent methods so far. As MNase-dependent nucleosome mapping has to employ limiting digests, resulting occupancy values (peak heights, trough depths) depend on the chosen digestion degree, which is difficult to control for or normalize for comparisons between independent experiments $[12,17]$. This obscures the comparison of nucleosome depletion between different conditions, like in vivo vesus in vitro chromatin, by MNase-based methods. Further yet, SGD chromatin can be prepared at arbitrary nucleosome densities, which also may affect the degree of nucleosome depletion over poly(dA:dT) tracts.

Here, we addressed these so far unresolved issues by using published absolute occupancy data obtained by MNase-independent DNA methylation footprinting (ODM-seq, [17]). This allows direct comparison of occupancies across conditions. We compared on the one hand ODM-seq data for S. cerevisiae in vivo chromatin, averaged over five replicates, as well as for SGD reconstituted chromatin along the $S$. cerevisiae genome at two different nucleosome densities with on the other hand published MNase-seq data of in vivo chromatin and for two replicate sets of SGD chromatin at three different densities each [62]. As ODM-seq reports nucleosome occupancy over the whole nucleosome length, we plotted MNase-seq data as $147 \mathrm{bp}$ extended dyads, too.

Classical in vivo +1 nucleosome-aligned composite plots showed the typical in vivo pattern of regularly spaced nucleosomal arrays downstream and the prominent nucleosome-free region (NFR) over the promoter region just upstream of the +1 nucleosome in both the ODM-seq and the MNase-seq data (Figure 1a-c). However, nucleosome depletion over promoters was less pronounced in SGD chromatin if measured by MNase-seq (Figure $1 \mathrm{~b}-\mathrm{c}$ ) and even less if measured by ODM-seq (Figure 1a). Nucleosome density had hardly an effect on the degree of nucleosome depletion in all cases. The corresponding traces of the three densities were virtually identical in the MNase-seq data. The differences between the replicates of MNase-seq data for SGD chromatin likely reflected the differences in MNase digestion degrees, which are notoriously diffcult to control exactly. As expected and shown before [17], absolute occupancy levels scaled with nucleosome density in the ODM-seq data (Figure 1a). The comparison of nucleosome densities in Figure 1a showed that the high nucleosome density in our genome-wide reconstitution system almost reached the in vivo density. 
(a)

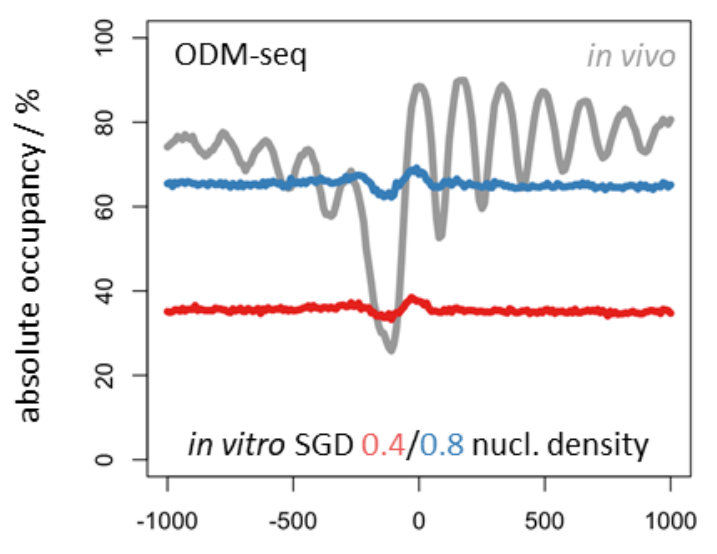

distance to in vivo +1 nucleosome / bp

(b)

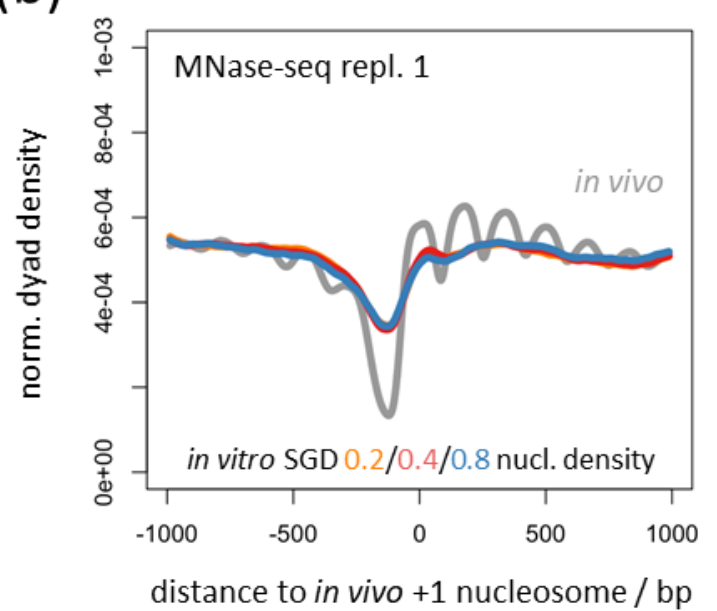

(c)

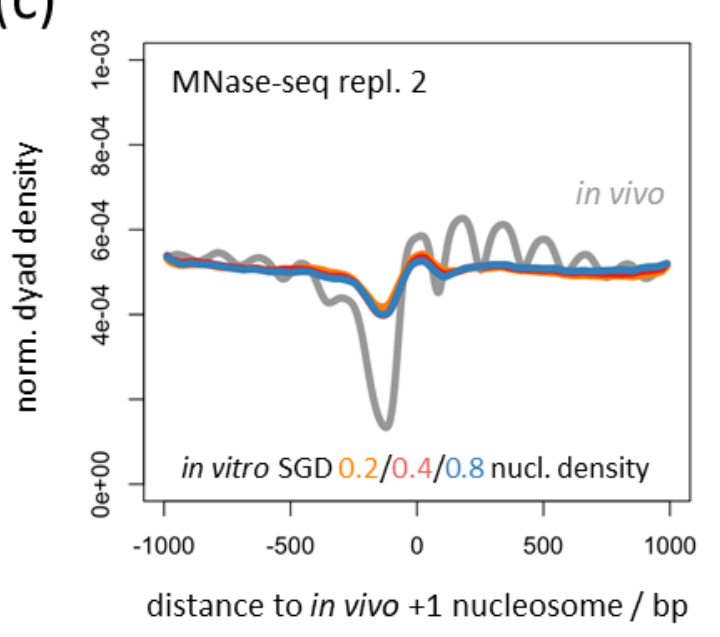

Figure 1. Monitoring nucleosome depletion over poly(dA:dT) tracts by DNA methylation footprinting (ODM-seq) shows that intrinsic nucleosome depletion in SGD chromatin is even less pronounced relative to the in vivo depletion in S. cerevisiae than previously seen by MNase-seq [38, 39, 63]. (a) Composite plots of absolute occupancy (ODM-seq data from [17]) for S. cerevisiae wild type cells (in vivo, grey, GSE141051) and for 0.4/0.8 nucleosome density reconstituted by SGD on an S. cerevisiae genomic library (in vitro SGD 0.4/0.8 nucl. density, red/blue, GSM4193222/GSM4193216, respectively) aligned at in vivo +1 nucleosome positions (annotation as in [64]). (b-c) Composite plots as in panel (a) but for two independent replicates of MNase-seq data from [62] for S. cerevisiae wild type cells (in vivo, grey, GSM4175394) and for 0.2/0.4/0.8 nucleosome density reconsti- 
tuted on an S. cerevisiae genomic library by SGD (in vitro SGD 0.2/0.4/0.8 nucl. density, orange/red/blue, replicate 1: GSM4175428/GSM4175429/GSM4175430, replicate 2: GSM4175803/GSM4175804/GSM4175805, respectively). MNase-seq data were plotted as $147 \mathrm{bp}$ extended dyads. Nucleosome density is quantified as described in [17, 62].

The classical plot type in Figure 1 shows the nucleosome depletion effect of poly(dA:dT) sequences only indirectly via their enrichment in $S$. cerevisiae promoters. This analysis is convoluted as there are promoters without poly $(\mathrm{dA}: \mathrm{dT})$ tracts and effects of binding competition with transcription factors and others shown to contribute to nucleosome depletion at promoters in addition to the assumed intrinsic mechanism over poly(dA:dT) tracts [2, 65-67]. To address the poly(dA:dT)-driven nucleosome depletion more directly, we binned all genomic poly(dA) tracts into three length categories (5 to 9 , 10 to 14 and more than $15 \mathrm{dA}$ in a row) and prepared composite plots of again the same ODM-seq and MNase-seq data as in Figure 1, but aligned at the centers of these poly(dA) tracts (Figure 2a-f). This clearly showed how nucleosome depletion over poly(dA) tracts, in vivo and for all nucleosome densities in vitro, increased with tract length. Note that composite plot traces became more rugged with decreasing number of instances as generally true. Depletion in vitro was very similar as in vivo if monitored by MNase-seq, which is the observation that suggested the intrinsic nucleosome depletion mechanism in the first place. However, this correlation was much less pronounced in ODM-seq data. This shows that the intrinsic nucleosome depletion over poly $(\mathrm{dA}: \mathrm{dT})$ tracts is inflated by the MNase digestion sequence bias, which preferentially removes nucleosomes that contain (dA:dT)-rich sequences. Nonetheless, long poly $(\mathrm{dA})$ tracts showed clear nucleosome depletion in vitro also by ODM-seq, which confirms that the intrinsic depletion over poly(dA:dT) tracts can be real. It is just much weaker than the in vivo depletion. 


\section{all poly $(\mathrm{dA})_{n} ; 5 \leq \mathrm{n} \leq 9$}

(a)

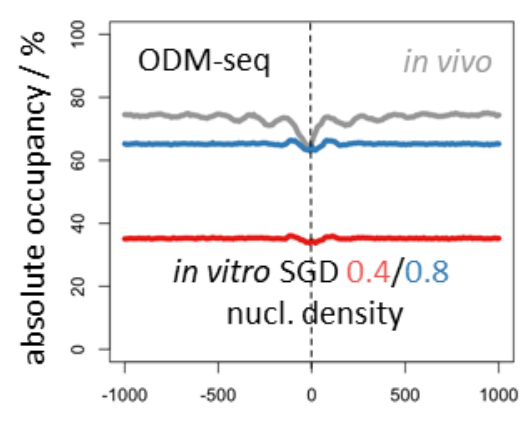

(b)

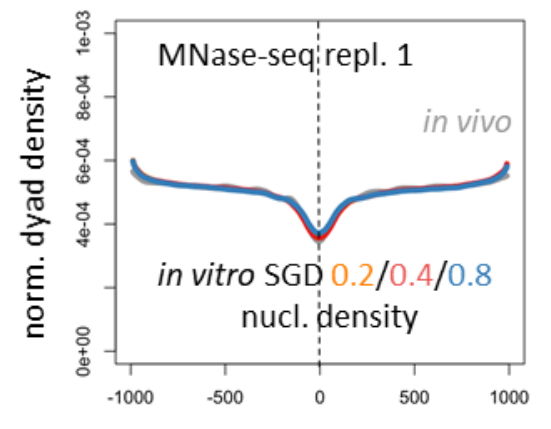

(c)

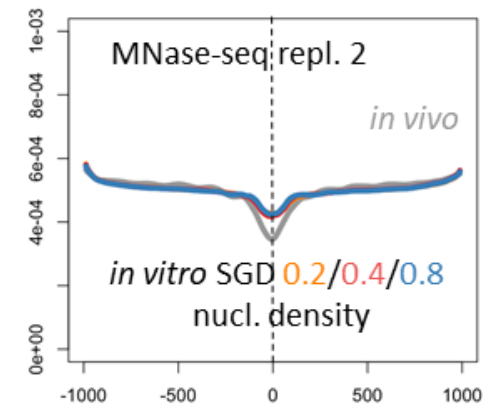

distance to center of poly (dA) tract / bp

all poly $(\mathrm{dA})_{n} ; 10 \leq \mathrm{n} \leq 14$

(d)

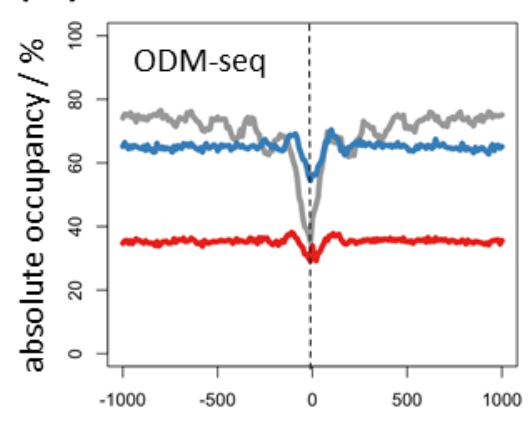

(e)

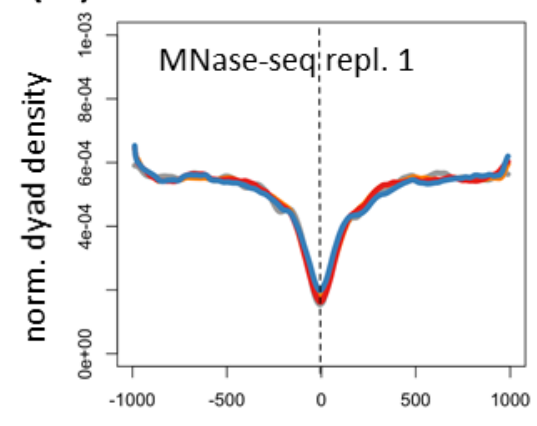

(f)

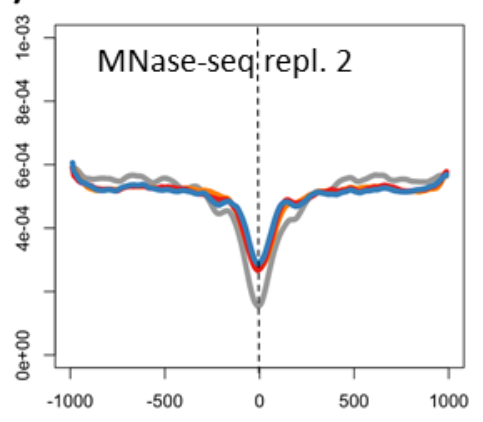

distance to center of poly $(\mathrm{dA})$ tract / bp

\section{all poly $(\mathrm{dA})_{n} ; \mathrm{n} \geq 15$}

(g)

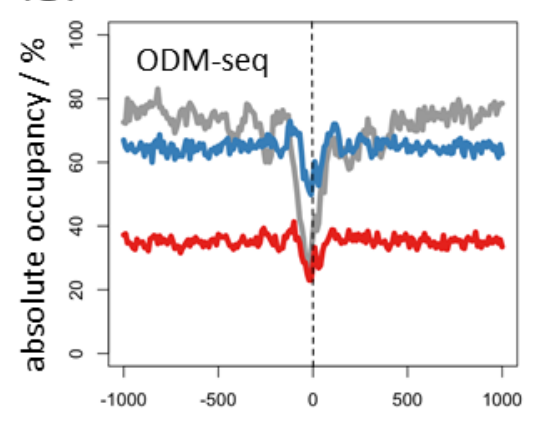

(h)

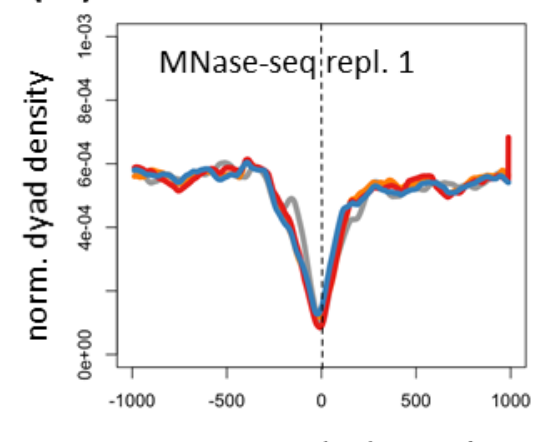

(i)

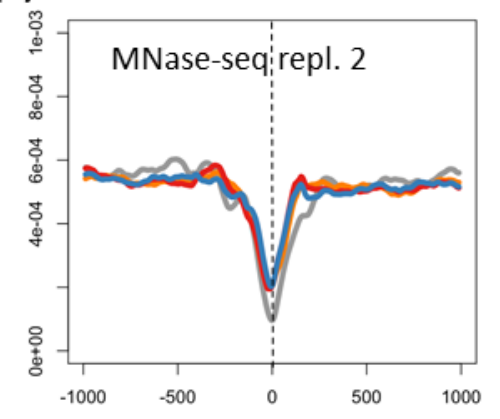

distance to center of poly(dA) tract / bp

Figure 2. Nucleosome depletion over poly $(\mathrm{dA} ; \mathrm{dT})$ tracts scales with tract length and seems similar in extent in vivo versus in vivo if monitored by MNase-seq but not by ODM-seq. Composite plots of same data as in Figure 1, but aligned at all poly(dA:dT) tracts in the S. cerevisiae genome of (a-c) five to nine (75,745 instances), (d-f) 10 to 14 (1,963 instances) and (g-i) 15 and more (303 instances) bp lengths. Strand orientation was taken into account by flipping the orientation for poly $(\mathrm{dA})$ tracts on the opposite strand. Vertical dashed lines mark the alignment points.

We wondered, if the much more pronounced discrepancy between nucleosome depletion in vivo versus in vitro over promoter regions (Figure 1) versus over poly(dA:dT) tracts (Figure 2) was due to some special feature of poly(dA:dT) tracts in promoters. Or, 
if it would actually be decreased if we focused only on promoters with poly $(\mathrm{dA}: \mathrm{dT})$ tracts, as the discrepancy may stem mainly from promoter regions without poly(dA:dT) tracts, which were encompassed in Figure 1 and where nucleosome depletion was due to some poly(dA:dT)-independent mechanism like factor binding competition. Therefore, we compared nucleosome depletion at all poly(dA:dT) tracts subdivided in promoter versus non-promoter regions (Figure 3). In ODM-seq data the disrepancy between in vivo and in vitro depletion was still especially pronounced at promoters (Figure 3a) and there was only a small increase in intrinsic nucleosome depletion in SGD chromatin over promoter versus non-promoter poly(dA:dT) tracts (Figure 3a versus 3b). In contrast, MNase-seq data showed similar relative differences between in vivo and in vitro depletion over poly(dA:dT) tracts in promoter versus non-promoter regions for both replicates, i.e., depletion in promoters was more pronounced to similar relative extents in vivo and in vitro (Figure 3c-f). Promoters are known to be especially enriched in poly(dA:dT) tracts, both in tract number as well as in average tract length. This affected nucleosome depletion in vivo as well as apparent intrinsic nucleosome depletion in vitro if monitored by MNase, probably due to the MNase-bias, but had a much smaller effect on intrinsic depletion as monitored by ODM-seq. 
promoters

(a)

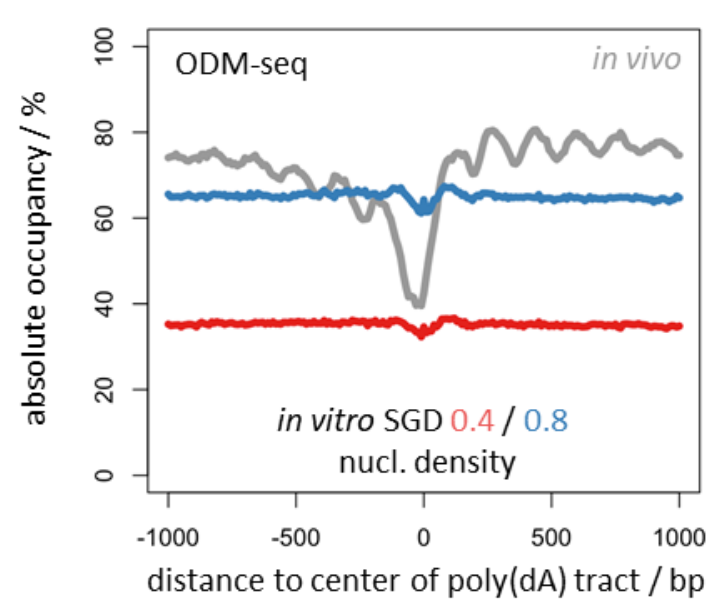

(c)

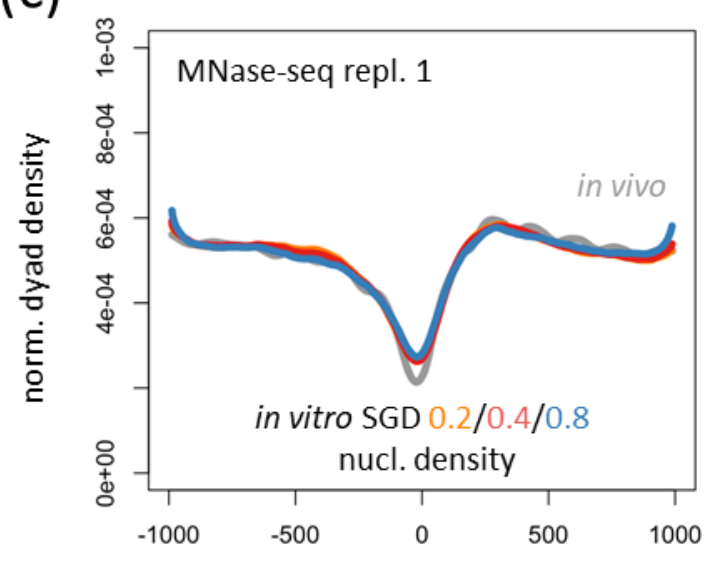

distance to center of poly(dA) tract / bp

(e)

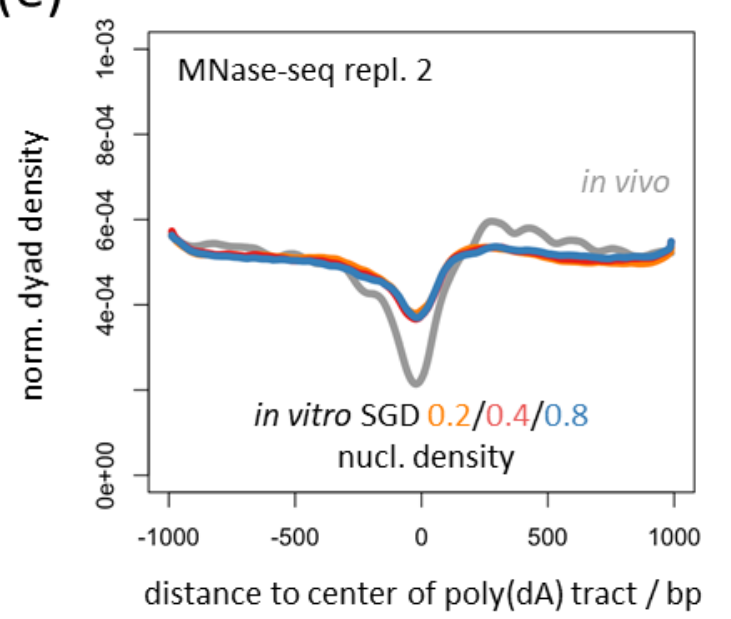

non-promoters

(b)

$\operatorname{poly}(\mathrm{dA})_{n} ; n \geq 5$

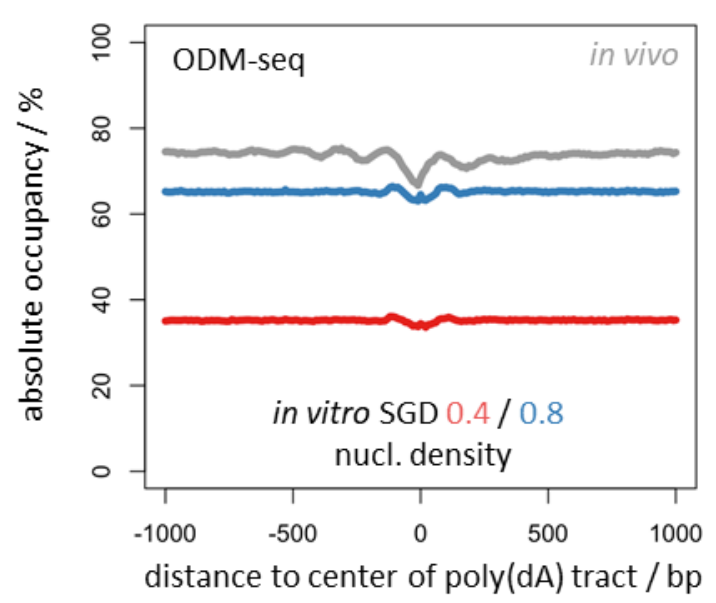

(d)

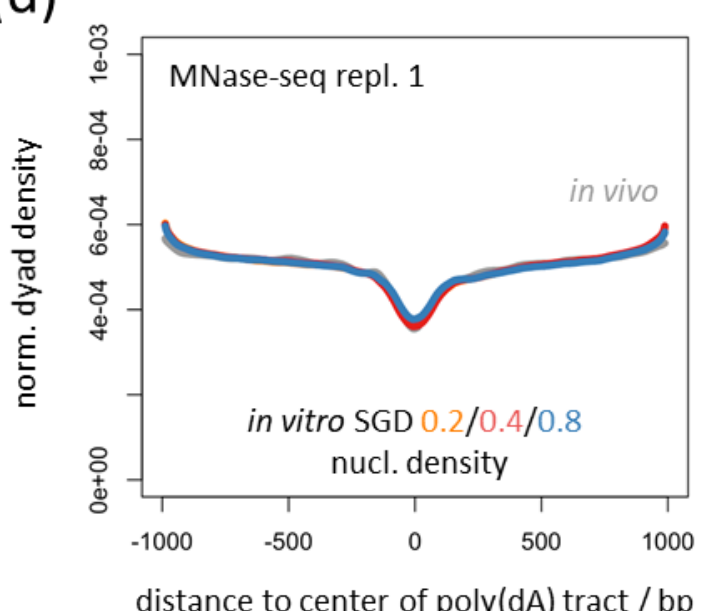

(f)

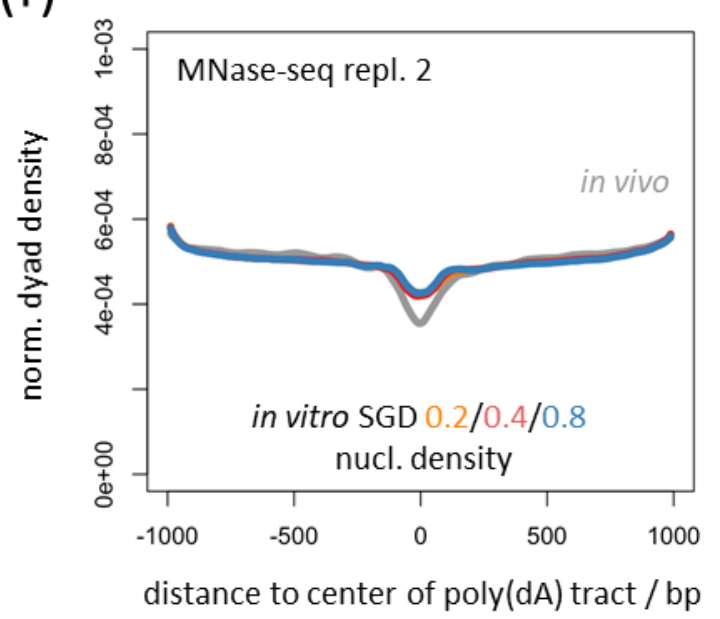

Figure 3. The difference between nucleosome depletion over poly(dA:dT) tracts in vivo versus in vitro is much more pronounced in promoter versus non-promoter regions if monitored by ODMseq than by MNase-seq. Composite plots of the same data as in Figure 1, but aligned at all poly(dA:dT) tracts with five or more bp lengths $(\mathbf{a}, \mathbf{c}, \mathbf{e})$ within $(8,802$ instances $)$ or $(\mathbf{b}, \mathbf{d}, \mathbf{f})$ outside $(69,300$ instances) of promoters (0 to $-250 \mathrm{bp}$ from transcription start sites [68]). Strand orientation was taken into account by flipping the orientation for poly $(\mathrm{dA})$ tracts on the opposite strand. 
Together, we confirm that there is poly $(\mathrm{dA})$-dependent nucleosome depletion by an intrinsic mechanism in vitro, and that there is a correlation with depletion over poly(dA) in vivo. However, the effects in vitro are so much weaker than in vivo if monitored by absolute occupancy in an MNase-independent way and especially in promoter regions enriched in poly(dA:dT) tracts (Figure 3a), that we wonder if anyone had ever drawn mechanistic conclusions from this correlation if MNase had not been the principal mapping tool.

Nucleosome depletion over poly(dA:dT) tracts is not an intrinsic default state but actively maintained

If poly $(\mathrm{dA}: \mathrm{dT})$ tracts excluded nucleosomes by a merely intrinsic mechanism, one may expect that they do so without further maintenance. Indeed, most poly $(\mathrm{dA}: \mathrm{dT})$ tracts in S. cerervisiae promoters are constitutively nucleosome-free and there is no viable mutant known where this is not the case [69, 70]. However, if the essential ATP dependent chromatin remodeling complex RSC and/or a certain class of essential sequence specific DNA binding proteins with binding sites in promoters, so called general regulatory factors (GRFs), like Reb1, Abf1 or Rap1, are ablated in conditional mutants, these NFRs start to fill up with nucleosomes [70-79]. This argues that promoter NFRs over poly(dA:dT) tracts are actively maintained by these factors and we suggest that this is the main reason why these factors are essential. Other non-essential remodelers, like the INO80 or the ISW2 complex, also participate in shaping promoter NFRs as NFR borders are affected in their respective deletion mutants $[70,74,80,81]$ and in in vitro reconstitutions $[39,62,64]$. Further, NFRs with or without poly $(\mathrm{dA}: \mathrm{dT})$ tracts are assembled into nucleosomes in the wake of replication and only later become nucleosome-free again [82-84].

The $S$. cerevisiae RSC remodeling complex preferentially evicts nucleosomes from poly(dA:dT) sequences

Active nucleosome depletion over poly(dA:dT) tracts could result from removing nucleosomes either in cis along the DNA (sliding) or in trans (disassembly). Turnover experiments in S. cerevisiae monitoring differentially labled histones demonstrated nucleosome removal in trans, especially at promoter NFRs [85-88]. Nucleosome disassembly in trans is catalyzed by only a subset of ATP dependent remodelers. Remodelers are classified according to sequence similarity among their ATPase motor subunits into four major families, the SWI/SNF, INO80, CHD and ISWI remodelers [56, 57, 89]. Only members of the SWI/SNF family show nucleosome disassembly activity in vitro. S. cerevisiae contains two SWI/SNF type remodelers, the SWI/SNF complex, after which the family was named, and the RSC complex [89]. Both are involved in promoter NFR or NDR (nucleosome depleted regions, see comment on terminology below) formation in vivo, but only RSC has an essential and pervasive role [70-79, 90]. Only RSC but not SWI/SNF was able to reconstitute in vivo-like NFRs in the context of a RSC-depleted whole cell extract in vitro [91] and RSC showed a preference for remodeling promoter versus genic nucleosomes in $e x$ vivo mini-chromosome circles [92]. This suggested that RSC may recognize some promoter feature. Indeed, RSC preferentially evicted nucleosomes from poly $(\mathrm{dA}: \mathrm{dT})$ tractcontaining DNA in mononucleosome assays in vitro [53].

\section{Directional nucleosome eviction from poly(dA:dT) tracts by RSC argues against the intrinsic but for an active nucleosome depletion mechanism}

The above arguments already point towards an active and targeted role for RSC in clearing nucleosomes from poly(dA:dT) tracts. Nonetheless, they could still be regarded as circumstancial and at least qualitatively compatible with the suggested intrinsic mechanism of nucleosome depletion. RSC may just implement the intrinsic sequence preferences. S. pombe in general [48] and S. cerevisiae promoters with ISW2-dependent nucleosome positioning [51] in particular may be examples where some other mechanism overrides intrinsic biophysics. However, there are more arguments that are based on bias and directionality of the poly $(\mathrm{dA}: \mathrm{dT})$-effect and clearly argue against the intrinsic mechanism. 
S. cerevisiae promoters show a biased distribution of poly $(\mathrm{dA}: \mathrm{dT})$ tracts such that poly $(\mathrm{dT})$ is often upstream of poly $(\mathrm{dA})$ on the strand that is continuous with the coding strand $[39,93,94]$. Such bias would not be expected to evolve in the context of a purely intrinsic mechanism. If in vivo nucleosome occupancy data is aligned at the center of poly(dA) tracts (Figures 2 and 3; [93]) or at the midpoint between pairwise combinations of 5'-poly(dA)-poly(dT), 5'-poly(dA)-poly(dA) or 5'-poly(dT)-poly(dA) tracts on the same strand [93], the NFRs are formed asymmetrically with a 5' or $3^{\prime}$ offset relative to poly(dA) or poly(dT), respectively. De Boer and Hughes [93] noted that the 5'-poly(dT)-poly(dA) pairwise arrangement thereby leads to mutual reinforcement of nucleosome displacement (from both sides towards the center of the paired tracts) and the strongest NFRs, which may explain why this evolved as the most common promoter organization in S. cerevisiae $[93,94]$. The same asymmetric NFR formation was also seen in mouse and human cells as well as in genome-wide chromatin reconstitutions with whole cell extracts and ATP [93], arguing for a role of remodelers. Again, such asymmetry or strandedness of the poly(dA:dT)-effect would not be expected for a purely intrinsic mechanism. For intrinsic nucleosome exclusion it should not matter which strand has the $\mathrm{dA}$ and which the $\mathrm{dT}$ bases. Indeed, SGD chromatin does not show a 5' offset of NFR formation over poly(dA) tracts (Figures 2 and 3; [93]), which also controls that this $5^{\prime}$ offset is not due to a biased distribution of poly $(\mathrm{dA}: \mathrm{dT})$ tracts in the vicinity of the alignment point. Even though the 5' offset was seen before in MNase-seq data [93], we note that it is mainly seen in ODMseq but not in MNase-seq data (Figure 2a, d, g and Figure 3a, b versus the other panels in Figures 2 and 3).

The directional nucleosome depletion over poly $(\mathrm{dA})$ tracts suggested that promoter sequences evolved so that they fit to a nucleosome depletion mechanism that reads out poly(dA:dT) tracts in a directional way, possibly via remodelers. Wu and Li [94] almost prophetically suggested (verbatim quote) "It is possible that G:C-capped poly(dA:dT) tracts may mark an initiation point for the remodeling activity of ATP dependent chromatin remodelers such as RSC." Indeed, this mechanism (without taking dG:dC-capping into account) was directly demonstrated in our genome-wide chromatin reconstitution system using purified components only [39]. SGD reconstitution of nucleosomes onto a genomic plasmid library representing essentially the whole $S$. cerevisiae genome preformed promoter NFRs according to the intrinsic nucleosome exclusion properties of poly(dA:dT) tracts. Incubation of this SGD chromatin with purified RSC and ATP widened these NFRs substantially and in a directional way leading to a $5^{\prime}$-offset relative to poly(dA) in promoters. Only RSC showed this directional nucleosome displacement, not the SWI/SNF, ISW1a, INO80 or ISW2 remodelers, which also controls against an MNasebias effect. Subsequent in vivo analyses that took poly(dA) tract directionality into account confirmed the corresponding directional effects on NFRs upon RSC ablation [75]. Finally, RSC was literally "caught in the act" while disassembling nucleosomes from S. cerevisiae promoter regions. RSC complexes were extracted from yeast nuclei by the CUT\&RUN technique and probed for histone content by anti-histone immunoprecipitation [95]. They were indeed associated with histones, especially the H2A histone variant H2A.Z, as well as with GRFs. This finding also resolved the debate around "fragile nucleosomes" (FN). FNs were operationally defined as particles of roughly nucleosome size that were detected in MNase-seq only at mild but not at more extensive MNase digestion degrees in yeast promoters [96]. Their nucleosomal nature was questioned as, for example, neither ChIPexo nor chemical mapping detected histone-DNA contacts in these regions [11, 14-16]. In the end, FNs apparently correspond to RSC complexes that are in the process of disassembling nucleosomes from (dA:dT)-rich regions. This underscores again that it is not the intrinsic default state of such regions to be nucleosome-free, but that there is a constitutive nucleosome assembly system at work, as also seen in the wake of replication, that is constantly and actively counteracted by the disassembly activity of RSC.

Remodelers are information processing hubs that turn DNA sequence information into nucleosome organization 
Together, this strongly argues that poly $(\mathrm{dA}: \mathrm{dT})$ tracts are specifically recognized by RSC so that RSC actively, preferentially and directionally displaces nucleosomes from these tracts. To be clear, this mechanism probably still entails the special intrinsic properties of poly(dA:dT) tracts as RSC reads them out while translocating along its tracking strand in the $3^{\prime}-5^{\prime}$ direction [97]. Therefore we still agree that the undebated intrinsic properties of poly(dA:dT) do play a role in nucleosome depletion in vivo. However, this depletion does not occur by an intrinsic mechanism, which entails only the interactions of DNA and histones as determinants of the resulting nucleosome organization. Such intrinsic mechanism seems unlikely in the light of the arguments given above and would definitely not show a directional effect with regard to the strandedness of poly(dA) tracts. Instead, the intrinsic poly $(\mathrm{dA}: \mathrm{dT})$ properties are actively implemented by extrinsic factors, for example, the RSC remodeler in $S$. cerevisiae.

In general, in vitro reconstitutions with SGD chromatin and purified remodelers demonstrated that remodelers like $S$. cerevisiae Chd1, RSC and INO80 are information processing hubs for nucleosome organization, with organization encompassing positioning as well as disassembly of nucleosomes. This means that they are able to read out DNA sequence and other information, e.g., distance to barriers like GRFs, and process this information together with their own input into remodeler-specific nucleosome organization $[39,64,98]$. The remodeler-specific information was conceptualized as a "remodeler ruler" $[62,99]$. Especially for the INO80 complex, the modules that make up the ruler and are involved in sensing and relaying DNA sequence information, in particular DNA shape features [100], were recently defined in structural terms [62, 64]. Also recently, the concept of intrinsic DNA bendability was revisited by a novel genome-scale cyclizibility assay for nucleosome-sized DNA fragments [101]. Also here, DNA sequence-intrinsic properties (bendability) correlated with in vivo nucleosome organization in S. cerevisiae, i.e., low bendability in NFRs and linkers versus high bendability in nucleosomes, so that a "DNA mechanical code" was suggested. Importantly, also these authors did not consider this an updated version of the purely intrinsic nucleosome positioning mechanism, but show, also with INO80 as example, that these differential DNA mechanics features are read out not just by the histone octamer but also by a remodeler.

All this fits to the earlier concept that different remodelers generate different nucleosome positioning on the same DNA sequence ("remodeler code" [102]) as they read out sequence information [103]. The DNA sequence information corresponds less so to classical sequence motifs but rather to the more redundantly implemented DNA shape and mechanics features. It should be noted that the classical intrinsic sequence preferences for nucleosome positioning as compared in competitive SGD reconstitutions represent rather static properties restricted to the contribution of DNA and histones. In contrast, remodelers may read out more dynamic DNA properties during twisting and bulging DNA in the context of the histone octamer and in the course of their ATP dependent remodeling mechanism $[60,104]$. Thereby remodelers add their own information that feeds into the resulting nucleosome organization and are not just "enzymes" that lubricate the equilibration of positions solely determined by the intrinsic interactions of DNA with histone octamers [105].

\section{Species-specific strategies for nucleosome depletion}

Nucleosome organization by remodeler-specific input offers a versatile and regulatable mechanism that explains in vivo observations beyond the best studied $S$. cerevisiae model. The at first confounding sequence features of nucleosome organization in $S$. pombe may simply mean that $S$. pombe remodelers evolved different ways of reading sequence features. For example, the $S$. pombe RSC complex lacks the Rsc3/30 subunits [106] that confer DNA binding interfaces for S. cerevisiae RSC [72]. Also other yeasts use poly(dA:dT) to varying degrees in their promoter organizations [2]. Further, poly(dA:dT) tracts are enriched in human promoters [107] but may not play the main role for nucleosome depletion there. Rather, human promoters that contain CpG islands, i.e., are rich in (dG:dC) corre- 
spond to the constitutively open poly $(\mathrm{dA}: \mathrm{dT})$-rich S. cerevisiae promoters [108]. The enrichment of (dG:dC), i.e., "nucleosome favouring" sequences in the intrinsic SGD-derived sense, is typical for promoters of human and other multicellular species $[109,110]$. In contrast to $S$. cerevisiae where the vast majority of the genome is constitutively expressed and most promoters developed an "open door policy" [111], only a minor genome fraction is expressed in each individual cell of a multicellular organism. Therefore, it appears that mechanisms for constitutive nucleosome depletion are less often hard-wired by directly remodeler-recognized sequences, but rather that regulatable mechanisms of indirect remodeler-recruitment, e.g., via transcription factor binding sites or by recruitment via histone-modifcations, open promoter chromatin on demand. Recently, an in-depth in vivo analysis of promoter nucleosome organization in two different human cell types, each with and without cell-type-specific gene induction stimuli, demonstrated cell type-specific, induction-specific and even very transient promoter opening [112].

\section{Afterthought on the terminology of nucleosome depletion}

For more than two decades, low nucleosome occupancy regions were mostly called nuclease hypersensitive sites or, more specifically, DNaseI hypersensitive sites [113-115]. This terminology is still in use and has the advantage of stating exactly the experimental observation, i.e., these regions are hypersensitive to nuclease digestion, and is therefore always appropriate as long as it fits the observation. Together with the first genome-scale mapping of nucleosome positions [20], Oliver Rando introduced the term nucleosomefree region (NFR). This term brings with it an interpretation of the observation, i.e., the low mapping signal is due to the absence of nucleosomes. Of note, this term encompasses two statments that are subject to debate: what is a nucleosome and does "free" really correspond to zero or just low occupancy? Accordingly, the designation as NFR has received criticism, for example, in the context of the discussion about FNs or non-canonical nucleosome particles in promoter regions [96, 116]. Many colleagues feel uneasy about the NFR term as it conveys a too extreme case of zero occupancy and they therefore prefer the more gradual connotation that comes with the term nucleosome depleted region (NDR), which is, to our perception, more pervasively used nowadays. Then again, there is the mechanistic NFR-versus-NDR-distinction by Frank Pugh, who underscores that at least in $S$. cerevisiae there are truely nucleosome-free promoter NFRs as measured by ChIP-exo and chemical mapping [14, 69, 117]. In contrast, NDRs in Pugh's terminology correspond to regulated regions, e.g., S. cerevisiae promoters, which are covered with nucleosomes in their repressed state and depleted of nucleosomes in the course of promoter activation. We follow the Pugh terminology here, but realize that this mechanistic distinction of NFR versus NDR seems to be less heard or understood than the quantitative distinction and is used less often. We are aware that observation and interpretation are epistemiologically entangled. In general, we advocate to first use observational terms and then to add the interpretation by qualifyers. (Nuclease) hypersensitive site is an operationally defined term that is probably easy to agree on. Such hypersensitive sites can then by qualified and quantified as constitutive, regulated, nucleosome-free or else.

Author Contributions: T.B. prepared the figures and commented on the manuscript, P.K. conceptualized and wrote the manuscript and acquired funding.

Funding: We are grateful for funding by the German Research Foundation (Deutsche Forschungsgemeinschaft, DFG, via SFB1064), which also funded the APC.

Data Availability Statement: Absolute nucleosome occupancy data used in Figures 1 to 3 are published in $[17,62]$ and available at GEO under the accession numbers given in the figure legends.

Acknowledgments: We acknowledge Michael R. Wolff for his help in plotting and interpreting the ODM-seq data. This article is dedicated to the memory of John Widom. Even though we argue against one of his favorite hypotheses, we deeply appreciate his biophysicist's mindset and approach that he brought to the chromatin field and mourn his untimely death. 


\section{References}

1. Field, Y.; Kaplan, N.; Fondufe-Mittendorf, Y.; Moore, I. K.; Sharon, E.; Lubling, Y.; Widom, J.; Segal, E., Distinct modes of regulation by chromatin encoded through nucleosome positioning signals. PLoS computational biology 2008, 4, (11), e1000216.

2. Tsankov, A. M.; Thompson, D. A.; Socha, A.; Regev, A.; Rando, O. J., The role of nucleosome positioning in the evolution of gene regulation. PLoS biology 2010, 8, (7), e1000414.

3. Segal, E.; Widom, J., Poly(dA:dT) tracts: major determinants of nucleosome organization. Current opinion in structural biology 2009, 19, (1), 65-71.

4. Lieleg, C.; Krietenstein, N.; Walker, M.; Korber, P., Nucleosome positioning in yeasts: methods, maps, and mechanisms. Chromosoma 2015, 124, (2), 131-51.

5. Jiang, C.; Pugh, B. F., Nucleosome positioning and gene regulation: advances through genomics. Nature reviews. Genetics 2009, 10, (3), 161-72.

6. Horz, W.; Altenburger, W., Sequence specific cleavage of DNA by micrococcal nuclease. Nucleic acids research 1981, 9, (12), 2643-58.

7. Dingwall, C.; Lomonossoff, G. P.; Laskey, R. A., High sequence specificity of micrococcal nuclease. Nucleic acids research 1981, 9, (12), 2659-73.

8. Caserta, M.; Agricola, E.; Churcher, M.; Hiriart, E.; Verdone, L.; Di Mauro, E.; Travers, A., A translational signature for nucleosome positioning in vivo. Nucleic acids research 2009, 37, (16), 5309-21.

9. Cockell, M.; Rhodes, D.; Klug, A., Location of the primary sites of micrococcal nuclease cleavage on the nucleosome core. Journal of molecular biology 1983, 170, (2), 423-46.

10. Weiner, A.; Hughes, A.; Yassour, M.; Rando, O. J.; Friedman, N., High-resolution nucleosome mapping reveals transcription-dependent promoter packaging. Genome research 2010, 20, (1), 90-100.

11. Chereji, R. V.; Ocampo, J.; Clark, D. J., MNase-Sensitive Complexes in Yeast: Nucleosomes and Non-histone Barriers. Molecular cell 2017, 65, (3), 565-577.e3.

12. Chereji, R. V.; Bryson, T. D.; Henikoff, S., Quantitative MNase-seq accurately maps nucleosome occupancy levels. Genome biology 2019, 20, (1), 198.

13. Rodriguez, J.; Tsukiyama, T., ATR-like kinase Mec1 facilitates both chromatin accessibility at DNA replication forks and replication fork progression during replication stress. Genes $\mathcal{E}$ development 2013, 27, (1), 74-86.

14. Rhee, H. S.; Bataille, A. R.; Zhang, L.; Pugh, B. F., Subnucleosomal structures and nucleosome asymmetry across a genome. Cell 2014, 159, (6), 1377-88.

15. Brogaard, K.; Xi, L.; Wang, J. P.; Widom, J., A map of nucleosome positions in yeast at base-pair resolution. Nature 2012, 486, (7404), 496-501.

16. Chereji, R. V.; Ramachandran, S.; Bryson, T. D.; Henikoff, S., Precise genome-wide mapping of single nucleosomes and linkers in vivo. Genome biology 2018, 19, (1), 19.

17. Oberbeckmann, E.; Wolff, M.; Krietenstein, N.; Heron, M.; Ellins, J. L.; Schmid, A.; Krebs, S.; Blum, H.; Gerland, U.; Korber, P., Absolute nucleosome occupancy map for the Saccharomyces cerevisiae genome. Genome research 2019, 29, (12), 1996-2009.

18. Stergachis, A. B.; Debo, B. M.; Haugen, E.; Churchman, L. S.; Stamatoyannopoulos, J. A., Single-molecule regulatory architectures captured by chromatin fiber sequencing. Science (New York, N.Y.) 2020, 368, (6498), 1449-1454.

19. Shipony, Z.; Marinov, G. K.; Swaffer, M. P.; Sinnott-Armstrong, N. A.; Skotheim, J. M.; Kundaje, A.; Greenleaf, W. J., Longrange single-molecule mapping of chromatin accessibility in eukaryotes. Nature methods 2020, 17, (3), 319-327.

20. Yuan, G. C.; Liu, Y. J.; Dion, M. F.; Slack, M. D.; Wu, L. F.; Altschuler, S. J.; Rando, O. J., Genome-scale identification of nucleosome positions in S. cerevisiae. Science (New York, N.Y.) 2005, 309, (5734), 626-30. 
21. Iyer, V.; Struhl, K., Poly(dA:dT), a ubiquitous promoter element that stimulates transcription via its intrinsic DNA structure. The EMBO journal 1995, 14, (11), 2570-9.

22. Struhl, K., Naturally occurring poly $(\mathrm{dA}-\mathrm{dT})$ sequences are upstream promoter elements for constitutive transcription in yeast. Proceedings of the National Academy of Sciences of the United States of America 1985, 82, (24), 8419-23.

23. Lee, W.; Tillo, D.; Bray, N.; Morse, R. H.; Davis, R. W.; Hughes, T. R.; Nislow, C., A high-resolution atlas of nucleosome occupancy in yeast. Nature genetics 2007, 39, (10), 1235-44.

24. Mavrich, T. N.; Ioshikhes, I. P.; Venters, B. J.; Jiang, C.; Tomsho, L. P.; Qi, J.; Schuster, S. C.; Albert, I.; Pugh, B. F., A barrier nucleosome model for statistical positioning of nucleosomes throughout the yeast genome. Genome research 2008, 18, (7), 1073-83.

25. Eaton, M. L.; Galani, K.; Kang, S.; Bell, S. P.; MacAlpine, D. M., Conserved nucleosome positioning defines replication origins. Genes $\mathcal{E}$ development 2010, 24, (8), 748-53.

26. Raveh-Sadka, T.; Levo, M.; Shabi, U.; Shany, B.; Keren, L.; Lotan-Pompan, M.; Zeevi, D.; Sharon, E.; Weinberger, A.; Segal, E., Manipulating nucleosome disfavoring sequences allows fine-tune regulation of gene expression in yeast. Nature genetics 2012, 44, (7), 743-50.

Hughes, A. L.; Jin, Y.; Rando, O. J.; Struhl, K., A functional evolutionary approach to identify determinants of nucleosome positioning: a unifying model for establishing the genome-wide pattern. Molecular cell 2012, 48, (1), 5-15.

Liu, G.; Zhao, H.; Meng, H.; Xing, Y.; Cai, L., A deformation energy model reveals sequence-dependent property of nucleosome positioning. Chromosoma 2021, 130, (1), 27-40.

29. Shrader, T. E.; Crothers, D. M., Effects of DNA sequence and histone-histone interactions on nucleosome placement. Journal of molecular biology 1990, 216, (1), 69-84.

30. Shrader, T. E.; Crothers, D. M., Artificial nucleosome positioning sequences. Proceedings of the National Academy of Sciences of the United States of America 1989, 86, (19), 7418-22.

31. Thåström, A.; Lowary, P. T.; Widom, J., Measurement of histone-DNA interaction free energy in nucleosomes. Methods (San Diego, Calif.) 2004, 33, (1), 33-44.

Luger, K.; Mader, A. W.; Richmond, R. K.; Sargent, D. F.; Richmond, T. J., Crystal structure of the nucleosome core particle at 2.8 A resolution. Nature 1997, 389, (6648), 251-60.

Korolev, N.; Vorontsova, O. V.; Nordenskiold, L., Physicochemical analysis of electrostatic foundation for DNA-protein interactions in chromatin transformations. Progress in biophysics and molecular biology 2007, 95, (1-3), 23-49.

Widom, J., Role of DNA sequence in nucleosome stability and dynamics. Quarterly reviews of biophysics 2001, 34, (3), $269-324$.

Drew, H. R., Can one measure the free energy of binding of the histone octamer to different DNA sequences by saltdependent reconstitution? Journal of molecular biology 1991, 219, (3), 391-2.

36.

Thåström, A.; Bingham, L. M.; Widom, J., Nucleosomal locations of dominant DNA sequence motifs for histone-DNA interactions and nucleosome positioning. Journal of molecular biology 2004, 338, (4), 695-709.

37. Lowary, P. T.; Widom, J., New DNA sequence rules for high affinity binding to histone octamer and sequence-directed nucleosome positioning. Journal of molecular biology 1998, 276, (1), 19-42.

38. Kaplan, N.; Moore, I. K.; Fondufe-Mittendorf, Y.; Gossett, A. J.; Tillo, D.; Field, Y.; LeProust, E. M.; Hughes, T. R.; Lieb, J. D.; Widom, J.; Segal, E., The DNA-encoded nucleosome organization of a eukaryotic genome. Nature 2009, 458, (7236), 362-6.

Krietenstein, N.; Wal, M.; Watanabe, S.; Park, B.; Peterson, C. L.; Pugh, B. F.; Korber, P., Genomic Nucleosome Organization Reconstituted with Pure Proteins. Cell 2016, 167, (3), 709-721.e12.

40. Zhang, Y.; Moqtaderi, Z.; Rattner, B. P.; Euskirchen, G.; Snyder, M.; Kadonaga, J. T.; Liu, X. S.; Struhl, K., Intrinsic histoneDNA interactions are not the major determinant of nucleosome positions in vivo. Nature structural $\mathcal{E}$ molecular biology 2009, $16,(8), 847-52$.

41. Flaus, A.; Owen-Hughes, T., Dynamic properties of nucleosomes during thermal and ATP-driven mobilization. Molecular and cellular biology 2003, 23, (21), 7767-79. 
42. Dyer, P. N.; Edayathumangalam, R. S.; White, C. L.; Bao, Y.; Chakravarthy, S.; Muthurajan, U. M.; Luger, K., Reconstitution of nucleosome core particles from recombinant histones and DNA. Methods in enzymology 2004, 375, $23-44$.

43. Andrews, A. J.; Luger, K., A coupled equilibrium approach to study nucleosome thermodynamics. Methods in enzymology 2011, 488, 265-85.

Andrews, A. J.; Luger, K., Nucleosome structure(s) and stability: variations on a theme. Annual review of biophysics 2011, 40 , 99-117.

Struhl, K.; Segal, E., Determinants of nucleosome positioning. Nature structural \& molecular biology 2013, 20, (3), 267-73. Segal, E.; Widom, J., What controls nucleosome positions? Trends in genetics : TIG 2009, 25, (8), 335-43.

Lantermann, A. B.; Straub, T.; Stralfors, A.; Yuan, G. C.; Ekwall, K.; Korber, P., Schizosaccharomyces pombe genome-wide nucleosome mapping reveals positioning mechanisms distinct from those of Saccharomyces cerevisiae. Nature structural $\mathcal{E}$ molecular biology 2010, 17, (2), 251-7.

48. Moyle-Heyrman, G.; Zaichuk, T.; Xi, L.; Zhang, Q.; Uhlenbeck, O. C.; Holmgren, R.; Widom, J.; Wang, J. P., Chemical map of Schizosaccharomyces pombe reveals species-specific features in nucleosome positioning. Proceedings of the National Academy of Sciences of the United States of America 2013, 110, (50), 20158-63.

49. Segal, E.; Fondufe-Mittendorf, Y.; Chen, L.; Thastrom, A.; Field, Y.; Moore, I. K.; Wang, J. P.; Widom, J., A genomic code for nucleosome positioning. Nature 2006, 442, (7104), 772-8.

Wippo, C. J.; Krstulovic, B. S.; Ertel, F.; Musladin, S.; Blaschke, D.; Sturzl, S.; Yuan, G. C.; Horz, W.; Korber, P.; Barbaric, S., Differential cofactor requirements for histone eviction from two nucleosomes at the yeast PHO84 promoter are determined by intrinsic nucleosome stability. Molecular and cellular biology 2009, 29, (11), 2960-81.

51. Whitehouse, I.; Rando, O. J.; Delrow, J.; Tsukiyama, T., Chromatin remodelling at promoters suppresses antisense transcription. Nature 2007, 450, (7172), 1031-5.

Losa, R.; Omari, S.; Thoma, F., Poly(dA).poly(dT) rich sequences are not sufficient to exclude nucleosome formation in a constitutive yeast promoter. Nucleic acids research 1990, 18, (12), 3495-502.

53. Lorch, Y.; Maier-Davis, B.; Kornberg, R. D., Role of DNA sequence in chromatin remodeling and the formation of nucleosome-free regions. Genes \& development 2014, 28, (22), 2492-7.

54. Bao, Y.; White, C. L.; Luger, K., Nucleosome core particles containing a poly(dA.dT) sequence element exhibit a locally distorted DNA structure. Journal of molecular biology 2006, 361, (4), 617-24.

55. Tran, Q. H.; Unden, G., Changes in the proton potential and the cellular energetics of Escherichia coli during growth by aerobic and anaerobic respiration or by fermentation. European journal of biochemistry 1998, 251, (1-2), 538-43.

56. Clapier, C. R.; Iwasa, J.; Cairns, B. R.; Peterson, C. L., Mechanisms of action and regulation of ATP-dependent chromatinremodelling complexes. Nature reviews. Molecular cell biology 2017, 18, (7), 407-422.

Harada, B. T.; Hwang, W. L.; Deindl, S.; Chatterjee, N.; Bartholomew, B.; Zhuang, X., Stepwise nucleosome translocation by RSC remodeling complexes. eLife 2016, 5.

Deindl, S.; Hwang, W. L.; Hota, S. K.; Blosser, T. R.; Prasad, P.; Bartholomew, B.; Zhuang, X., ISWI remodelers slide nucleosomes with coordinated multi-base-pair entry steps and single-base-pair exit steps. Cell 2013, 152, (3), 442-52.

Zhou, C. Y.; Johnson, S. L.; Gamarra, N. I.; Narlikar, G. J., Mechanisms of ATP-Dependent Chromatin Remodeling Motors. Annual review of biophysics 2016, 45, 153-81.

61. Andrews, A. J.; Chen, X.; Zevin, A.; Stargell, L. A.; Luger, K., The histone chaperone Nap1 promotes nucleosome assembly by eliminating nonnucleosomal histone DNA interactions. Molecular cell 2010, 37, (6), 834-42.

Oberbeckmann, E.; Niebauer, V.; Watanabe, S.; Farnung, L.; Moldt, M.; Schmid, A.; Cramer, P.; Peterson, C. L.; Eustermann, S.; Hopfner, K. P.; Korber, P., Ruler elements in chromatin remodelers set nucleosome array spacing and phasing. Nature communications 2021, in press. 
63. Zhang, Z.; Wippo, C. J.; Wal, M.; Ward, E.; Korber, P.; Pugh, B. F., A packing mechanism for nucleosome organization reconstituted across a eukaryotic genome. Science (New York, N.Y.) 2011, 332, (6032), 977-80.

64. Oberbeckmann, E.; Krietenstein, N.; Niebauer, V.; Wang, Y.; Schall, K.; Moldt, M.; Straub, T.; Rohs, R.; Hopfner, K. P.; Korber, P.; Eustermann, S., Genome information processing by the INO80 chromatin remodeler positions nucleosomes. Nature communications 2021, in press.

65. Ozonov, E. A.; van Nimwegen, E., Nucleosome free regions in yeast promoters result from competitive binding of transcription factors that interact with chromatin modifiers. PLoS computational biology 2013, 9, (8), e1003181.

66. Kharerin, H.; Bai, L., Thermodynamic modeling of genome-wide nucleosome depleted regions in yeast. PLoS computational biology 2021, 17, (1), e1008560.

67. Tsankov, A.; Yanagisawa, Y.; Rhind, N.; Regev, A.; Rando, O. J., Evolutionary divergence of intrinsic and trans-regulated nucleosome positioning sequences reveals plastic rules for chromatin organization. Genome research 2011, $21,(11), 1851-62$.

68. Xu, Z.; Wei, W.; Gagneur, J.; Perocchi, F.; Clauder-Munster, S.; Camblong, J.; Guffanti, E.; Stutz, F.; Huber, W.; Steinmetz, L. M., Bidirectional promoters generate pervasive transcription in yeast. Nature 2009, 457, (7232), 1033-7.

69. Rossi, M. J.; Kuntala, P. K.; Lai, W. K. M.; Yamada, N.; Badjatia, N.; Mittal, C.; Kuzu, G.; Bocklund, K.; Farrell, N. P.; Blanda, T. R.; Mairose, J. D.; Basting, A. V.; Mistretta, K. S.; Rocco, D. J.; Perkinson, E. S.; Kellogg, G. D.; Mahony, S.; Pugh, B. F., A high-resolution protein architecture of the budding yeast genome. Nature 2021, 592, (7853), 309-314.

70. van Bakel, H.; Tsui, K.; Gebbia, M.; Mnaimneh, S.; Hughes, T. R.; Nislow, C., A compendium of nucleosome and transcript profiles reveals determinants of chromatin architecture and transcription. PLoS genetics 2013, 9, (5), e1003479.

71. Parnell, T. J.; Huff, J. T.; Cairns, B. R., RSC regulates nucleosome positioning at Pol II genes and density at Pol III genes. The EMBO journal 2008, 27, (1), 100-10.

Badis, G.; Chan, E. T.; van Bakel, H.; Pena-Castillo, L.; Tillo, D.; Tsui, K.; Carlson, C. D.; Gossett, A. J.; Hasinoff, M. J.; Warren, C. L.; Gebbia, M.; Talukder, S.; Yang, A.; Mnaimneh, S.; Terterov, D.; Coburn, D.; Li Yeo, A.; Yeo, Z. X.; Clarke, N. D.; Lieb, J. D.; Ansari, A. Z.; Nislow, C.; Hughes, T. R., A library of yeast transcription factor motifs reveals a widespread function for Rsc3 in targeting nucleosome exclusion at promoters. Molecular cell 2008, 32, (6), 878-87.

73. Hartley, P. D.; Madhani, H. D., Mechanisms that specify promoter nucleosome location and identity. Cell 2009, 137, (3), 44558.

74. Kubik, S.; Bruzzone, M. J.; Challal, D.; Dreos, R.; Mattarocci, S.; Bucher, P.; Libri, D.; Shore, D., Opposing chromatin remodelers control transcription initiation frequency and start site selection. Nature structural E molecular biology 2019, 26, (8), 744-754.

Kubik, S.; O'Duibhir, E.; de Jonge, W. J.; Mattarocci, S.; Albert, B.; Falcone, J. L.; Bruzzone, M. J.; Holstege, F. C. P.; Shore, D., Sequence-Directed Action of RSC Remodeler and General Regulatory Factors Modulates +1 Nucleosome Position to Facilitate Transcription. Molecular cell 2018, 71, (1), 89-102.e5.

76. Challal, D.; Barucco, M.; Kubik, S.; Feuerbach, F.; Candelli, T.; Geoffroy, H.; Benaksas, C.; Shore, D.; Libri, D., General Regulatory Factors Control the Fidelity of Transcription by Restricting Non-coding and Ectopic Initiation. Molecular cell 2018, 72, (6), 955-969.e7.

77. Ganguli, D.; Chereji, R. V.; Iben, J. R.; Cole, H. A.; Clark, D. J., RSC-dependent constructive and destructive interference between opposing arrays of phased nucleosomes in yeast. Genome research 2014, 24, (10), 1637-49.

Klein-Brill, A.; Joseph-Strauss, D.; Appleboim, A.; Friedman, N., Dynamics of Chromatin and Transcription during Transient Depletion of the RSC Chromatin Remodeling Complex. Cell reports 2019, 26, (1), 279-292.e5.

Rawal, Y.; Chereji, R. V.; Qiu, H.; Ananthakrishnan, S.; Govind, C. K.; Clark, D. J.; Hinnebusch, A. G., SWI/SNF and RSC cooperate to reposition and evict promoter nucleosomes at highly expressed genes in yeast. Genes $\mathcal{E}$ development 2018, 32, (9-10), 695-710. 
80. Qiu, H.; Biernat, E.; Govind, C. K.; Rawal, Y.; Chereji, R. V.; Clark, D. J.; Hinnebusch, A. G., Chromatin remodeler Ino80C acts independently of H2A.Z to evict promoter nucleosomes and stimulate transcription of highly expressed genes in yeast. Nucleic acids research 2020, 48, (15), 8408-8430.

81. Yen, K.; Vinayachandran, V.; Batta, K.; Koerber, R. T.; Pugh, B. F., Genome-wide nucleosome specificity and directionality of chromatin remodelers. Cell 2012, 149, (7), 1461-73.

82. Ramachandran, S.; Henikoff, S., Transcriptional Regulators Compete with Nucleosomes Post-replication. Cell 2016, 165, (3), 580-92.

83. Fennessy, R. T.; Owen-Hughes, T., Establishment of a promoter-based chromatin architecture on recently replicated DNA can accommodate variable inter-nucleosome spacing. Nucleic acids research 2016, 44, (15), 7189-203.

84. Vasseur, P.; Tonazzini, S.; Ziane, R.; Camasses, A.; Rando, O. J.; Radman-Livaja, M., Dynamics of Nucleosome Positioning Maturation following Genomic Replication. Cell reports 2016, 16, (10), 2651-2665.

85. Schermer, U. J.; Korber, P.; Horz, W., Histones are incorporated in trans during reassembly of the yeast PHO5 promoter. Molecular cell 2005, 19, (2), 279-85.

86. Dion, M. F.; Kaplan, T.; Kim, M.; Buratowski, S.; Friedman, N.; Rando, O. J., Dynamics of replication-independent histone turnover in budding yeast. Science (New York, N.Y.) 2007, 315, (5817), 1405-8.

Rufiange, A.; Jacques, P. E.; Bhat, W.; Robert, F.; Nourani, A., Genome-wide replication-independent histone H3 exchange occurs predominantly at promoters and implicates H3 K56 acetylation and Asf1. Molecular cell 2007, 27, (3), 393-405.

88. Jamai, A.; Imoberdorf, R. M.; Strubin, M., Continuous histone H2B and transcription-dependent histone H3 exchange in yeast cells outside of replication. Molecular cell 2007, 25, (3), 345-55.

89. Flaus, A.; Martin, D. M.; Barton, G. J.; Owen-Hughes, T., Identification of multiple distinct Snf2 subfamilies with conserved structural motifs. Nucleic acids research 2006, 34, (10), 2887-905.

90. Cairns, B. R.; Lorch, Y.; Li, Y.; Zhang, M.; Lacomis, L.; Erdjument-Bromage, H.; Tempst, P.; Du, J.; Laurent, B.; Kornberg, R. D., RSC, an essential, abundant chromatin-remodeling complex. Cell 1996, 87, (7), 1249-60.

91. Wippo, C. J.; Israel, L.; Watanabe, S.; Hochheimer, A.; Peterson, C. L.; Korber, P., The RSC chromatin remodelling enzyme has a unique role in directing the accurate positioning of nucleosomes. The EMBO journal 2011, 30, (7), 1277-88.

92. Lorch, Y.; Griesenbeck, J.; Boeger, H.; Maier-Davis, B.; Kornberg, R. D., Selective removal of promoter nucleosomes by the RSC chromatin-remodeling complex. Nature structural \& molecular biology 2011, 18, (8), 881-5. de Boer, C. G.; Hughes, T. R., Poly-dA:dT tracts form an in vivo nucleosomal turnstile. PloS one 2014, 9, (10), e110479.

$\mathrm{Wu}, \mathrm{R}$; $\mathrm{Li}, \mathrm{H}$., Positioned and G/C-capped poly(dA:dT) tracts associate with the centers of nucleosome-free regions in yeast promoters. Genome research 2010, 20, (4), 473-84.

Brahma, S.; Henikoff, S., RSC-Associated Subnucleosomes Define MNase-Sensitive Promoters in Yeast. Molecular cell 2019, 73, (2), 238-249.e3.

96. Kubik, S.; Bruzzone, M. J.; Jacquet, P.; Falcone, J. L.; Rougemont, J.; Shore, D., Nucleosome Stability Distinguishes Two Different Promoter Types at All Protein-Coding Genes in Yeast. Molecular cell 2015, 60, (3), 422-34.

Saha, A.; Wittmeyer, J.; Cairns, B. R., Chromatin remodeling through directional DNA translocation from an internal nucleosomal site. Nature structural \& molecular biology 2005, 12, (9), 747-55.

98. Winger, J.; Bowman, G. D., The Sequence of Nucleosomal DNA Modulates Sliding by the Chd1 Chromatin Remodeler. Journal of molecular biology 2017, 429, (6), 808-822.

99. Yamada, K.; Frouws, T. D.; Angst, B.; Fitzgerald, D. J.; DeLuca, C.; Schimmele, K.; Sargent, D. F.; Richmond, T. J., Structure and mechanism of the chromatin remodelling factor ISW1a. Nature 2011, 472, (7344), 448-53.

100. Zhou, T.; Yang, L.; Lu, Y.; Dror, I.; Dantas Machado, A. C.; Ghane, T.; Di Felice, R.; Rohs, R., DNAshape: a method for the high-throughput prediction of DNA structural features on a genomic scale. Nucleic acids research 2013, 41, (Web Server issue), W56-62. 
101. Basu, A.; Bobrovnikov, D. G.; Qureshi, Z.; Kayikcioglu, T.; Ngo, T. T. M.; Ranjan, A.; Eustermann, S.; Cieza, B.; Morgan, M. T.; Hejna, M.; Rube, H. T.; Hopfner, K. P.; Wolberger, C.; Song, J. S.; Ha, T., Measuring DNA mechanics on the genome scale. Nature 2021, 589, (7842), 462-467.

102. Rippe, K.; Schrader, A.; Riede, P.; Strohner, R.; Lehmann, E.; Langst, G., DNA sequence- and conformation-directed positioning of nucleosomes by chromatin-remodeling complexes. Proceedings of the National Academy of Sciences of the United States of America 2007, 104, (40), 15635-40.

103. van Vugt, J. J.; de Jager, M.; Murawska, M.; Brehm, A.; van Noort, J.; Logie, C., Multiple aspects of ATP-dependent nucleosome translocation by RSC and Mi-2 are directed by the underlying DNA sequence. PloS one 2009, 4, (7), e6345. dependent chromatin remodelling by the INO80 complex. Nature 2018, 556, (7701), 386-390.

105. Korber, P., Active nucleosome positioning beyond intrinsic biophysics is revealed by in vitro reconstitution. Biochemical Society transactions 2012, 40, (2), 377-82.

106. Monahan, B. J.; Villen, J.; Marguerat, S.; Bahler, J.; Gygi, S. P.; Winston, F., Fission yeast SWI/SNF and RSC complexes show compositional and functional differences from budding yeast. Nature structural $\mathcal{E}$ molecular biology 2008, 15, (8), 873-80.

107. Ozsolak, F.; Song, J. S.; Liu, X. S.; Fisher, D. E., High-throughput mapping of the chromatin structure of human promoters. Nature biotechnology 2007, 25, (2), 244-8.

108. Haberle, V.; Lenhard, B., Promoter architectures and developmental gene regulation. Seminars in cell E developmental biology 2016, 57, 11-23.

109. Tillo, D.; Kaplan, N.; Moore, I. K.; Fondufe-Mittendorf, Y.; Gossett, A. J.; Field, Y.; Lieb, J. D.; Widom, J.; Segal, E.; Hughes, T. R., High nucleosome occupancy is encoded at human regulatory sequences. PloS one 2010, 5, (2), e9129.

110. Tompitak, M.; Vaillant, C.; Schiessel, H., Genomes of Multicellular Organisms Have Evolved to Attract Nucleosomes to Promoter Regions. Biophysical journal 2017, 112, (3), 505-511.

111. Morse, R. H., Transcription factor access to promoter elements. Journal of cellular biochemistry 2007, 102, (3), 560-70.

112. Oruba, A.; Saccani, S.; van Essen, D., Role of cell-type specific nucleosome positioning in inducible activation of mammalian promoters. Nature communications 2020, 11, (1), 1075.

113. Reinke, H.; Horz, W., Anatomy of a hypersensitive site. Biochimica et biophysica acta 2004, 1677, (1-3), $24-9$.

114. Elgin, S. C., Anatomy of hypersensitive sites. Nature 1984, 309, (5965), 213-4.

115. Wu, C., The 5' ends of Drosophila heat shock genes in chromatin are hypersensitive to DNase I. Nature 1980, 286, (5776), $854-60$

116. Jin, C.; Zang, C.; Wei, G.; Cui, K.; Peng, W.; Zhao, K.; Felsenfeld, G., H3.3/H2A.Z double variant-containing nucleosomes mark 'nucleosome-free regions' of active promoters and other regulatory regions. Nature genetics 2009, 41, (8), 941-5. 